\title{
Laboratory protocols for testing the efficacy of commercial pit latrine additives
}

\author{
KM Foxon*, S Mkhize, M Reddy and CA Buckley \\ Pollution Research Group, School of Chemical Engineering, University of KwaZulu-Natal, Durban 4041, South Africa
}

\begin{abstract}
There is considerable national interest in the use of commercial microbially derived products for controlling the rate of accumulation of the contents of pit latrines. Manufacturers claim that some of these products can reduce accumulation rates, prevent the pit from ever filling up, or even result in decreases in pit contents volume. Prior to this research, there have been no scientific publications that have conclusively supported or refuted these claims.

This project undertook to perform reproducible laboratory tests that would quantify the effect of commercial pit latrine additive products. Protocols were developed and tested on a range of different commercial products sold for their ability to control the rate of accumulation of pit latrine contents. The effect of commercial additives on mass loss from VIP sludge in $300 \mathrm{~g}$ honey jars was compared to mass loss from similar units subjected to no treatment and treatment with water.

The purpose of these experiments was to separate and quantify the effect of micro-organisms or enzymes originating from commercial pit latrine additives from the effect of natural processes within the pit latrine sludge (including digestion by micro-organisms in the VIP sludge and dehydration) and the effects of other actions associated with treatment, such as the addition of water.

Results indicated that insignificant mass loss occurred in all anaerobic test units, while significant mass loss occurred in all other test units. However, there was no statistically significant difference between any of the different treatments in the aerobic units. Investigation of analytical data from the test units indicated that mass loss in aerobic units was due to a combination of dehydration through evaporation of moisture and biological stabilisation processes, and that the latter were not significantly enhanced by the addition of commercial pit latrine products. It was concluded that there was no evidence to support claims that pit latrine additives could extend the life of a pit latrine.
\end{abstract}

Keywords: additives, pit latrines, VIP

\section{Introduction}

A well-designed and operated VIP has reduced fly and odour problems and therefore improved health benefits compared to rudimentary pits (Franceys et al., 1992; Cotton et al., 1995; Bester and Austin, 2000) but poor degradation in most pits results in a build-up of noxious and potentially hazardous material that must ultimately be removed at significant cost to either the householder or local authority or both (Still, 2002).

In a rural setting pits are generally allowed to fill to within a certain proximity to the top of the pit (e.g. $300 \mathrm{~mm}$ ) and then covered over, a new pit is dug nearby, and the superstructure moved or rebuilt. A period of stabilisation may be allowed before pit contents are removed and the pit reused. In a peri-urban densely populated setting, full pits are either emptied by pumping out the contents (or in the case of solid contents, digging out) since alternative sanitation facilities or sites for a new pit are not usually available (Cotton et al., 1995; Still, 2002). Clearly, extending the life of the pit by reducing the rate of accumulation of material in the pit could result in substantial savings in pit maintenance.

There are a number of proprietary products available that are marketed for their ability to reduce pit contents, odour or

This paper was originally presented at the 2008 Water Institute of Southern Africa (WISA) Biennial Conference, Sun City, South Africa, 18-22 May 2008

* To whom all correspondence should be addressed.

5ir +2731 2601490; fax: +2731 2601118;

e-mail: foxonk@ukzn.ac.za fly problems. Independent scientific evidence of their efficacy is scarce, although there is a vast body of anecdotal evidence that suggests that they have the ability to significantly reduce pit contents, fly and odour problems. Equally, a number of informal studies have suggested that there is no significant benefit to the use of these additives over the addition of water or some essentially inert additive (in effect, a placebo). In many instances, municipalities and other service providers are hesitant to sanction the use of pit latrine additives as they have no scientific basis for choosing one product over another, and are concerned that trials with these products may lead to an expectation among communities that the products will be used forthwith. This in turn could lead to a situation where political pressure results in the application of expensive products that may or may not have any significant benefit. It is generally felt that a scientific explanation of the mechanism of pit latrine additives, and proof of their efficacy would provide the authorities with the ability to rationally assess the cost-effectiveness of implementing programmes for treating pits with additives.

A preliminary trial into the effect of pit latrine additives was conducted in the field as part of a wider study into processes in pit latrines (Buckley et al., 2008). Testing of pit latrine additives in the field was found to produce inconclusive results due to the difficulty of obtaining representative measurements of any condition or property within the pit and the lack of control of the test site. It was apparent that many of the observations drawn from field studies relate to the condition and ownership of the pit rather than the biological processes (or lack thereof) occurring within the pit (Buckley et al., 2008). The field study concluded that it is necessary to have an independently managed, well-controlled 
testing protocol through which an assessment of the effect of the additive on the pit latrine contents can be made. This paper presents the results of a laboratory study into the effects of commercial pit-latrine products on pit-latrine contents.

\section{Published studies on the use of pit-latrine additives}

A WRC project (Taljaard et al., 2003) evaluated the ability of microbial or microbially-derived products to treat pit-latrine contents. This study had two experimental parts: firstly, samples of faeces were incubated under aerobic conditions at $22^{\circ} \mathrm{C}$. A volume ratio of approximately $2: 5$ faeces to pit-latrine additive solution was used. This is several orders of magnitude greater than the volume ratios that would be used in the field. Twelve different pit-latrine additives were tested. The faeces were combined with sterilised water to make a paste and added in a thin layer on the bottom of each respirometer flask. To the paste was added the calculated dosage of bio-supplement and a small amount of a nutrient solution. Samples were agitated throughout the $5 \mathrm{~d}$ experiment. The conditions created in the respirometer were therefore ideal for micro-biological degradation of the faecal material: a high ratio of micro-organism originating from the bio-supplement to food was supplied; no inhibiting effects that might be caused by presence of urine in a pit were experienced, an unlimited oxygen supply with little mass transfer resistance was provided and agitation ensured that good contact between bio-supplement and faeces was obtained. Under these conditions, COD and total suspended solids (TSS) removal (calculated from average sample concentrations measured before and after incubation) in control vessels was found to be higher in units treated with pit-latrine additive than in control units. Rigorous statistical analysis of the data was not presented. Products that caused improved COD reduction appeared to usually result in improved TSS reduction. These results indicate that certain of the products at the dosages applied may have been able to significantly increase COD and TSS removal rates over those which occur naturally. However the dosages used in these experiments were far above those that could be used in practice.

Two of the products that had been shown to be effective in removing COD and TSS were used to treat two blocks of pit latrines on a farm. Pits were watered on a daily basis to simulate urine addition. A third block was treated with the same amount of water as the other two blocks, but no bio-supplement was added. Researchers tried to prevent the labourers from using any of these pits during the course of the study. The pits were open (did not have pedestals or squat hole covers) and did not have vent pipes. Some reduction in pit contents was seen in the two blocks where the toilets were treated with pitlatrine additives, and none was recorded for the control blocks; however, the volume reduction was very little (a maximum of $22 \mathrm{~cm}$ over a period of 3 months when no additional solid material was being added). The authors of this study suggested that pit-latrine additives are able to reduce pit contents (Taljaard et al., 2003).

In a second pit-latrine additive study, a pit-latrine additive consisting of spore-forming non-pathogenic bacteria was dosed to 4 pit latrines weekly for four weeks (Jere et al., 1998). This study concluded that the treatment reduced the height of pit contents. However, there was no control against which the results could be compared. The authors cautiously concluded that the additives showed promise for reducing filling rates. However, the results did not indicate the contribution to the decrease in pit contents height of the method of application (injection under pressure through a perforated tube into the pit contents).
In summary, although some work has been undertaken to test the efficacy of commercial pit-latrine additives on controlling filling rates, the work reported does not provide sufficient evidence to prove that additives containing micro-organisms significantly increase the rate of degradation of pit-latrine contents; however, micro-organisms from pit-latrine additives are clearly able to digest pit-latrine contents.

\section{Development of a laboratory protocol for testing pit-latrine additives}

The hypotheses to be tested by the laboratory protocol were:

- That through the biological action of micro-organisms present in pit-latrine additives, the overall mass of a sample of pit-latrine contents could be reduced at a faster rate than could be achieved by natural degradative processes mediated by micro-organisms present in the pit-latrine contents; or

- That the addition of pit-latrine additives had no significant effect on the rate of mass loss or the rate of change of composition of samples of pit-latrine contents as determined by total COD, soluble COD, moisture and solids content measurements.

\section{Materials and methods}

The laboratory protocol consisted of a test in which a sample of VIP contents, sampled from the surface of the pit beneath the pit pedestal, was mixed and divided into sub-samples of known mass (approximately $300 \mathrm{~g}$ each) that were placed in $300 \mathrm{~m} \ell$ screw-top honey jars. A number of treatments were applied to these units. The following terminology has been defined:

- A unit was a single honey jar containing approximately 300 $\mathrm{g}$ of VIP contents

- A treatment was a set of 3 or 5 units within a trial that have all been set up in the same way (i.e. all units that have been treated with a fixed dose of Additive A.)

- A trial consisted of treatments (including reference and control treatments) that have been set up from a single wellmixed sample of VIP contents.

- Aerobic units were open with no hindrance to the movement of air between bulk air supply and the top of the VIP contents in the units

- Anaerobic units were closed, limiting access of air to the VIP contents in each unit.

The dosing rate of additives was determined as mass (or volume) additive per surface area of the pit $\left[\mathrm{g} / \mathrm{m}^{2}\right]$ and the same dosing rate was applied to the honey jars. Tests were performed in three or five replicates. Two reference treatments (or controls) were included for comparative purposes, namely no addition of water or chemicals (control); or addition of water (water reference).

The mass of the honey jars was measured when empty, immediately after filling and at intervals of approximately $3 \mathrm{~d}$ for between 27 and $46 \mathrm{~d}$ after commencement of the experiment.

COD concentration [g COD/g sample], moisture content [g $\mathrm{H}_{2} \mathrm{O} / \mathrm{g}$ sample] and total solids content $[\mathrm{g} \mathrm{TS} / \mathrm{g}$ sample] were determined on each test unit at the beginning and end of the experiment.

From these data, the following values were calculated:

- Rate of mass loss in each unit

- Extent of COD reduction in each unit

- Extent of moisture loss in each unit. 
Mass loss rates, COD concentration and moisture content were compared between treatments with additives and similar measures obtained for control units and water reference units.

\section{Results}

Two trials were undertaken in this study. In the $1^{\text {st }}$ trial, the effect of aerobic and anaerobic conditions was tested. In the $2^{\text {nd }}$ trial, the relative humidity of the air supply for aerobic tests was increased to reduce evaporation.

\section{Trial 1}

In Trial 1, the protocols were tested under both aerobic and anaerobic conditions. The difference in conditions was achieved by setting up 2 sets of test units, where screw top lids were tightly fitted to the honey jars of the anaerobic set and the aerobic set were left open. All treatments were incubated for $46 \mathrm{~d}$ in a fume cupboard.

Under anaerobic conditions, only a very small fraction of mass was lost from any of the test units. No water reference units were constructed for the anaerobic set. Figure 1 shows the average rate of mass loss from each of the units in the anaerobic set of Trial 1. These values were calculated using standard linear regression techniques.

Figure 1 shows a large distribution of mass loss rates across all the units. No significant change in height was observed for samples incubated under anaerobic conditions. The overall rate of mass loss from anaerobic units in Trial 1 was $0.036 \mathrm{~kg} / \mathrm{m}^{2}$ surface area/d. No clear trend could be identified in examination of the mass loss rates. The overall variation in mass loss rate between treatments was similar, with only Product E showing a mass loss rate that was systematically higher for all 5 treatments. It was noted that although the relative variation between rates calculated in the anaerobic test appeared to be large, the absolute variation in rates was actually larger in the aerobic tests (data presented in detail below).

These results imply that for all of the treatments considered under anaerobic conditions, the amount of biological activity is insignificant (all treatments lost less than $3 \%$ of their original mass under the conditions tested).

In contrast to the results obtained under anaerobic conditions, when the test units were left open to the air (i.e. without lids), higher mass loss rates were recorded. The average rate of mass loss across all aerobic units in Trial 1 was $0.80 \mathrm{~kg} / \mathrm{m}^{2}$ surface area/d (Fig. 2).

The average rate of mass loss in aerobic units was more than 22 times greater than the equivalent rate of mass loss in anaerobic units. This result clearly indicates that the processes that facilitate mass reduction in pit-latrine samples require exposure to air. These include dehydration and biological conversion of organic material to gases.

Figure 2 shows that there was very little difference between any of the treatments. Once again a distribution of mass loss rates was observed between units within a treatment. None of the treatments showed a systematic improvement in mass loss

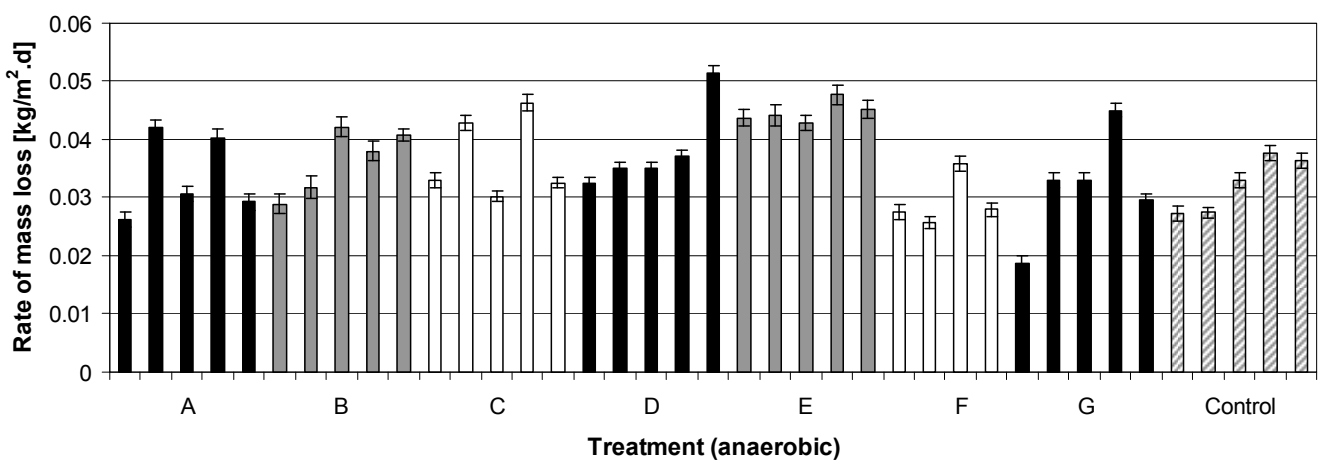

Figure 1

Trial 1: Anaerobic set (honey jars sealed with screw-on lids). Average rate of mass loss after $46 \mathrm{~d}$ of incubation at $25^{\circ} \mathrm{C}$. Seven pit-latrine additives ( $A$ to $G$ ) were tested in 5 replicates. Control indicates samples incubated without additional water or additive. Error bars indicate $95 \%$ confidence interval on the rate of mass loss.

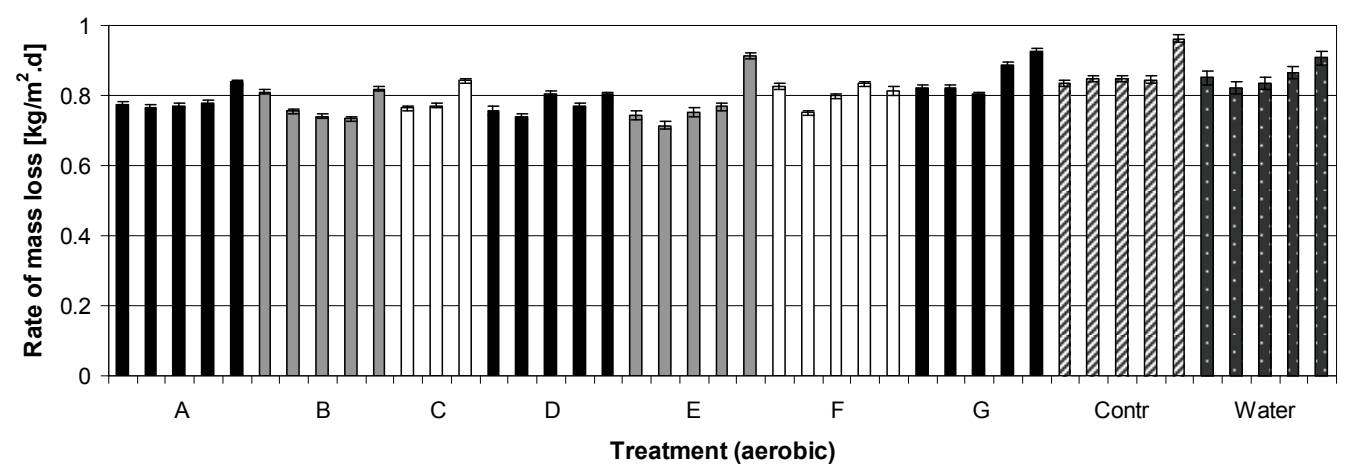

Figure 2

Trial 1: Aerobic set (open honey jars). Average rate of mass loss after 46 days of incubation at $25^{\circ} \mathrm{C}$. Seven pit-latrine additives ( $A$ to $G$ ) were tested in 5 replicates. Contr indicates samples incubated without additional water or additive. Water indicates samples to which tap water only was added. Error bars indicate $95 \%$ confidence interval on the rate of mass loss. 

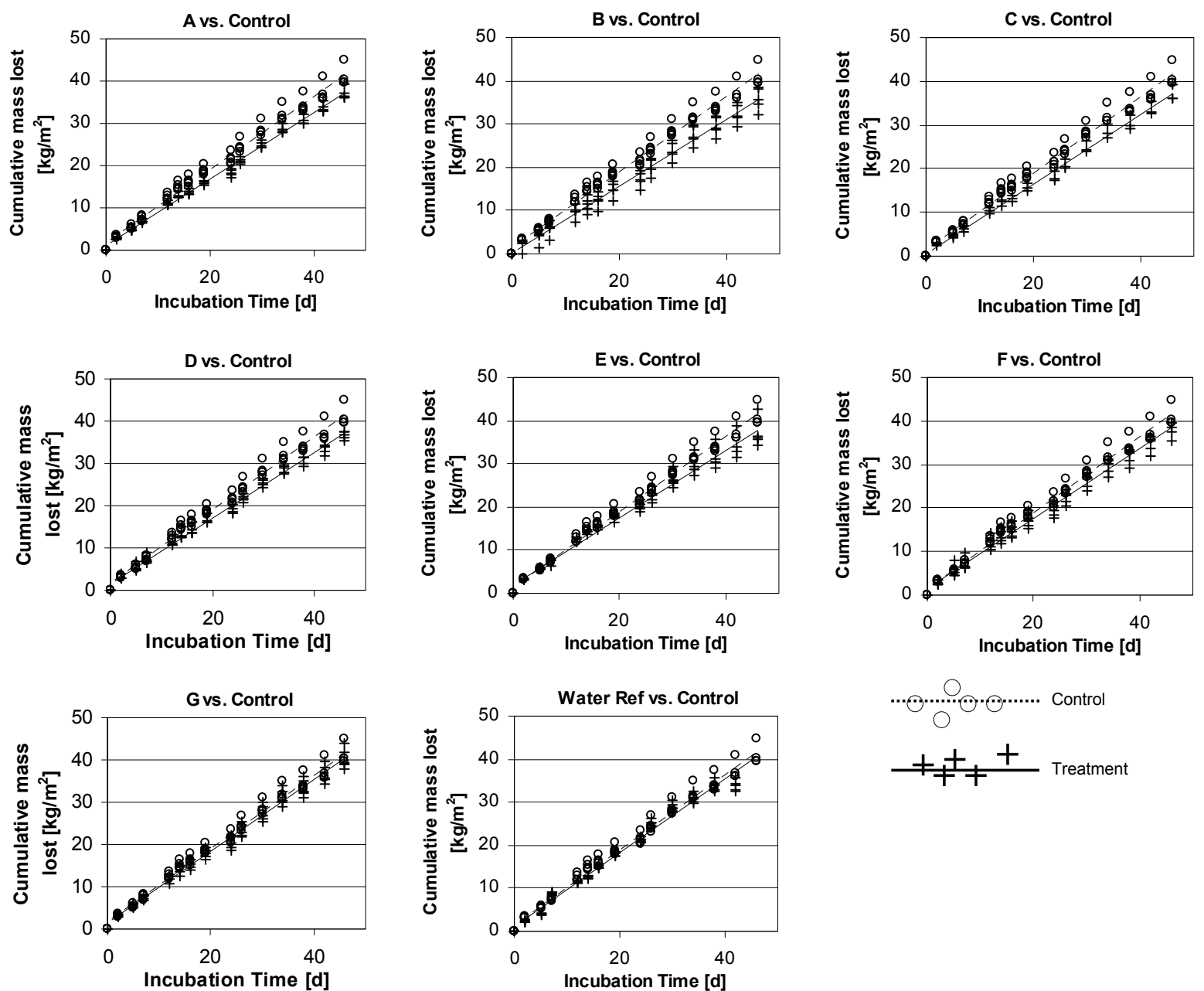

Figure 3

Trial 1 (Aerobic units): Cumulative mass lost from treatments $A$ to $G$ and water reference compared to similar mass loss data from control units. Each graph shows data from 5 replicates of each treatment.

rate across all units within the treatment compared to either the control or the water reference units. These relationships are shown in greater detail in Fig. 3, which shows data for each additive treatment and water reference units compared to equivalent data from the control treatment. Each data set was fitted with a straight line by linear regression. For this trial, it was observed that the regressed line for the controls fell above the regressed line for each of the other treatments, i.e. the amount of mass loss appeared to be consistently greater in control units than in units treated with commercial additives. However, it was found that the difference was not statistically significant for any of the combinations shown. Thus it was concluded that for Trial 1, proprietary additives A to $\mathrm{G}$ did not have a significant effect (either positive or negative) on the rate of natural mass loss processes that occurred within samples of pit-latrine contents.

Measurements of moisture and COD concentration were made in the bulk sample before the start of the trial, and on samples taken from the surface of 3 of 5 replicates from each aerobic treatment at the end of the trial. Results of these analyses are presented in Table 1.

The initial moisture content was found to be $0.83 \mathrm{~g} \mathrm{H}_{2} \mathrm{O} / \mathrm{g}$ wet sample. This value is extremely high, higher than any other moisture content measured in pit-latrine contents in the course of the larger project of which this study was a part (Buckley et
TABLE 1

Trial 1 (aerobic units): Moisture and COD concentration measured in the bulk sample of pit-latrine contents used in trial 1 before the start of the trial (initial), and from the surface of the aerobic units at the end of the aerobic trial (final). Final values are presented as a $95 \%$ confidence interval of the mean ( $n=$ number of observations)

\begin{tabular}{|l|c|c|}
\hline & $\begin{array}{c}\text { Moisture } \\
\text { [ } \mathbf{g ~ H}_{\mathbf{2}} \mathbf{O} / \mathbf{g} \text { wet sample] }\end{array}$ & $\begin{array}{c}\text { COD } \\
\text { [mg COD/g dry sample] }\end{array}$ \\
\hline Initial & 0.83 & 1100 \\
\hline Final & $0.60-0.64(\mathrm{n}=27)$ & $450-550(\mathrm{n}=27)$ \\
\hline
\end{tabular}

al., 2008), and similar to maximum values recorded in faeces. As a result the COD content reported on a dry basis was also very high (1 $100 \mathrm{mg} \mathrm{COD} / \mathrm{g}$ sample). Insufficient replicates were performed to gain an indication of the variance of these values.

Statistical analysis of the data showed that there was no significant difference between final moisture and COD concentration from any of the treatments and the control. These results indicate that although both moisture and COD content decreased, the amount of moisture loss and COD reduction was independent of the treatment. The lack of replication in the initial moisture content and COD concentration data means that it 


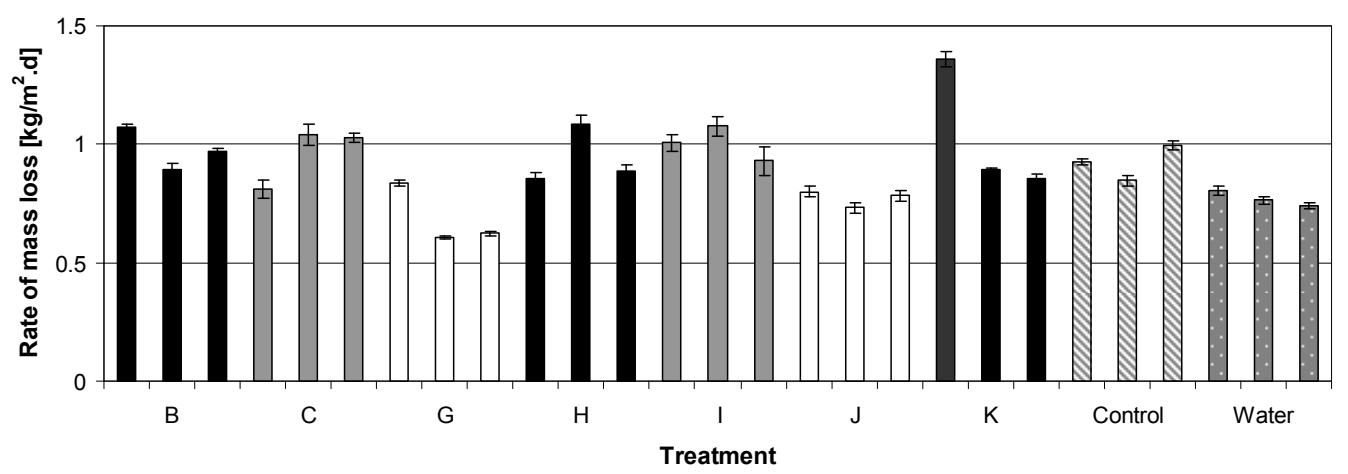

Figure 4

Trial 2: Aerobic set (honey jars sealed with screw-on lids). Average rate of mass loss after $27 d$ of incubation at $25^{\circ} \mathrm{C}$. Seven pit-latrine additives were tested in 3 replicates. Control indicates samples incubated without additional water or additive. Water indicates samples to which tap water only was added. Error bars indicate 95\% confidence interval on the rate of mass loss. is not possible to make any conclusions about the magnitude of moisture and COD reduction with any certainty. Furthermore, since only surface samples were analysed (and the surface is expected to have undergone a greater degree of dehydration and biological degradation than the buried bulk), it was also not possible to perform a mass balance to determine how much of the overall mass loss could be attributed to moisture loss.

Moisture loss through dehydration may have had a significant effect on the interpretation of the results of Trial 1 under aerobic conditions. Thus it was proposed that the test protocol should be modified to supply test units with air saturated with moisture in an attempt to reduce the overall rate of dehydration. By reducing the mass loss due to dehydration, the contribution of biological degradation to mass loss could be more clearly identified.

\section{Trial 2}

Trial 2 followed the same basic protocol as Trial 1 using fresh additives that had been recently acquired from a number of suppliers, including some of the same brands tested in Trial 1, and a number of new brands. Three of the additive brands tested in Trial 1 were used again in Trial 2 while the remaining 4 had not been previously tested. To reduce the rate of dehydration from test units, the humidity in the fume cupboard was increased.

Only aerobic units were set up since it had been shown that overall rates of mass loss were insignificant under anaerobic conditions. Only 3 replicates were used for each treatment, and the trial was terminated after $27 \mathrm{~d}$.

Figure 4 presents the rate of mass loss from each of the units in Trial 2. It was observed that the variance in rate of mass loss was higher for Trial 2 than Trial 1; the overall variance of slopes in the aerobic set of Trial 1 was $3.1 \times 10^{-3} \mathrm{~kg} / \mathrm{m}^{2} \cdot \mathrm{d}$, while that for Trial 2 was $2.7 \times 10^{-2} \mathrm{~kg} / \mathrm{m}^{2} \cdot \mathrm{d}$. There were two mathematical reasons for this difference; firstly, only 3 replicates were used in Trial 2, while 5 were used in Trial 1, and the length of the experiment in Trial 2 was shorter than in Trial 1, resulting in fewer data points (10 vs. 15 for each unit). However, examination of Fig. 2 and Fig. 4 suggests that the variation in mass loss rate due to dehydration or biological activity may also have been greater in Trial 2 compared to Trial 1.

The overall rate of mass loss from all units in Trial 2 was $0.90 \mathrm{~kg} / \mathrm{m}^{2}$ surface area/day. Comparison of the mass loss rates from the control sets of Trial 1 and 2 indicated that although the data from Trial 2 appeared to indicate that the mass loss occurred at a higher rate in Trial 2 than in Trial 1, the difference was not statistically significant (Welch test, $\mathrm{P}=0.48$ ). Similarly, by considering the entire set of regressed mass loss rates across all aerobic units in Trial 1 and Trial 2 despite an apparent increase in mass loss rate, the difference was not statistically significant (Welch test, $\mathrm{P}=0.47$ ). Thus it may be concluded that for these two trials, the source of pit-latrine contents did not have a significant effect on the results. However, this conclusion may have been different if the average mass loss rate for each unit in Trial 2 could have been determined with greater certainty (i.e. with a smaller confidence interval on the reported value). This conclusion also does not take into account differences in prevailing conditions of the test, specifically the increased relative humidity in the fume cupboard in Trial 2.

Figure 5 (next page) presents cumulative mass loss data from treatments with commercial pit-latrine additives and the water references compared to the control units for Trial 2 . In all cases, the average regression line for the treatment is similar to that of the control. However, unlike the observation in Trial 1 , the regression lines of certain of the treatments $(\mathrm{B}, \mathrm{H}$ and $\mathrm{I})$ were above those of the control. To test whether the apparent differences were statistically significant or not, the regression slope data (including the calculated variances of the regression slopes) were subjected to a Welch test to determine the probability that the mean slopes of the treatment and control slopes were significantly different. In all comparisons, the Welch test returned $\mathrm{P}>0.45$; i.e. the probability that the slopes were not significantly different was greater than $45 \%$ and therefore none of the treatments resulted in significantly greater mass loss rates than observed in the control (A difference is only considered to be statistically significant if the probability that the two values are the same is less than 5\%).

These results confirm the findings of Trial 1 that treatment of pit-latrine contents with commercial additives under the conditions used in the laboratory protocols resulted in no significant difference to the rate of mass loss from the samples.

\section{TABLE 2}

Trial 2: Moisture and COD concentration measured in the bulk sample of pit-latrine contents used in trial 2 before the start of the trial (initial), and from the surface of the aerobic units at the end of the aerobic trial (final). Final values are presented as a $95 \%$ confidence interval of the mean ( $n=$ number of observations)

\begin{tabular}{|l|c|c|}
\hline & $\begin{array}{c}\text { Moisture } \\
\text { [g H} \mathbf{~}_{2} \mathbf{O} / \mathbf{g} \text { wet sample] }\end{array}$ & $\begin{array}{c}\text { COD } \\
\text { [mg COD/g dry sample] }\end{array}$ \\
\hline Initial & 0.78 & 730 \\
\hline Final & $0.71-0.78(\mathrm{n}=14)$ & $630-950(\mathrm{n}=14)$ \\
\hline
\end{tabular}

A further interesting result was that the rate of mass loss from Trial 2 was similar to that of Trial 1 despite the fact that the rate of dehydration was less as a result of the air supply being saturated with water. Examination of moisture data (Table 2) showed that the final moisture content in the units at the end of Trial 2 was significantly higher than those recorded in Trial 1 , and in fact similar to the initial value. Thus it appears that 

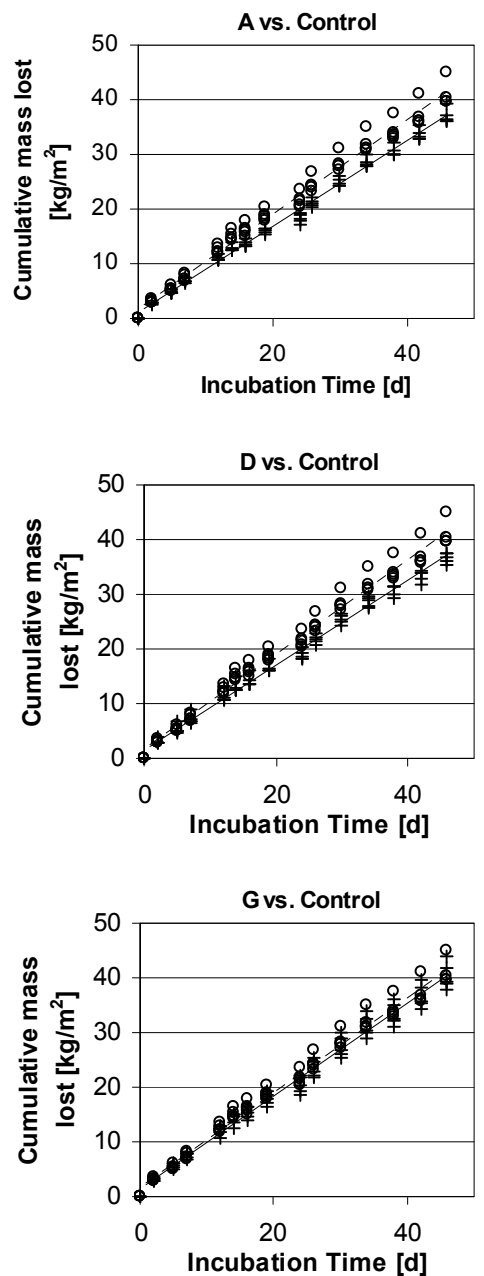
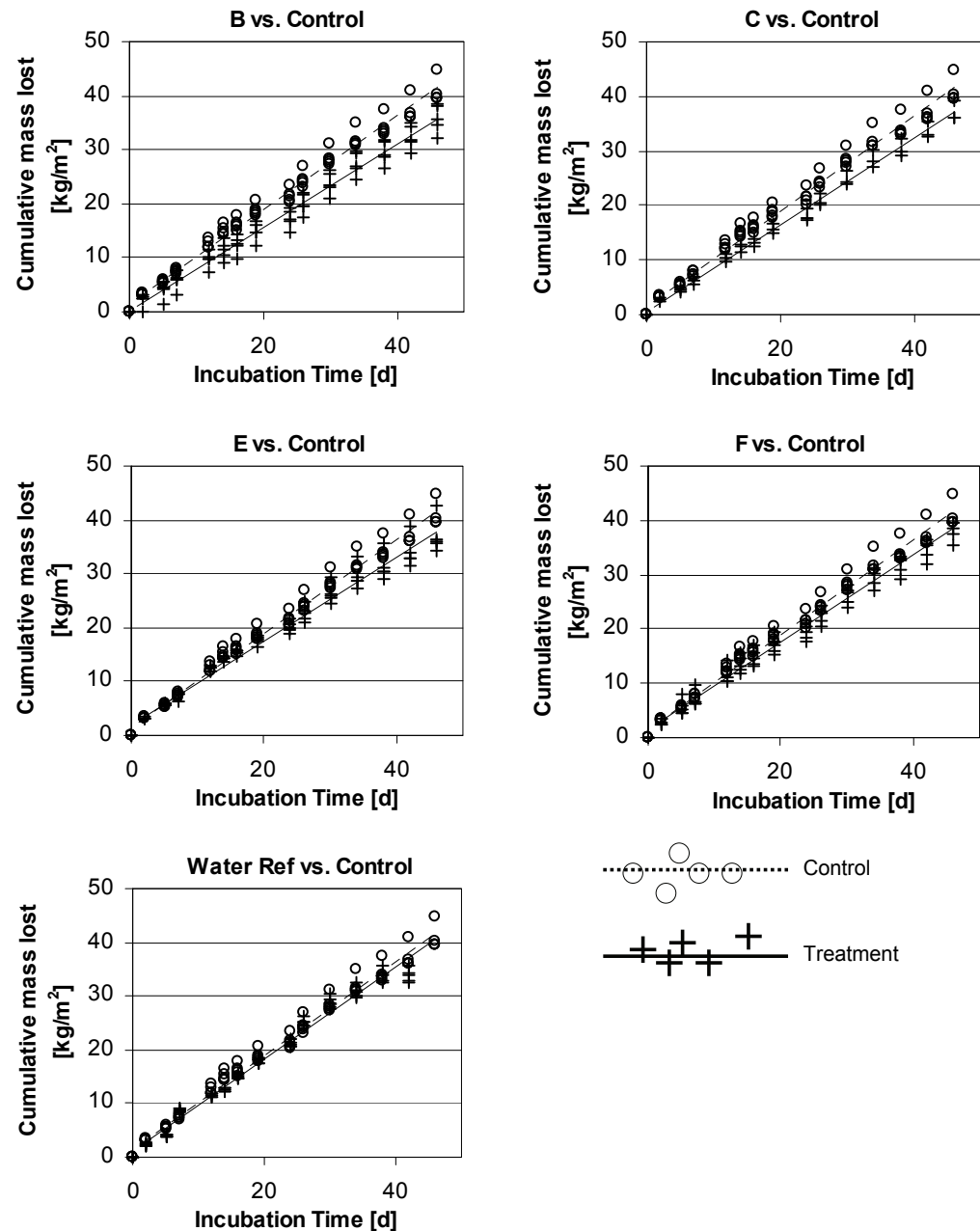

Figure 5

Trial 2: Cumulative mass lost from treatments $B, C, G$, and $H$ to $K$ and water references compared to similar mass loss data from control units. Each graph shows data from 3 replicates of each treatment. supplying saturated air to the units resulted in a reduction in the rate of dehydration, and it must therefore be concluded that a significant fraction of the overall mass loss in the units in Trial 2 was due to biological conversion of organic material to carbon dioxide. Furthermore, in Trial 1, it would appear that some of the mass loss observed was due to dehydration. It follows that the rate of biological activity in Trial 2 must have been higher than in Trial 1 if the overall mass loss rates were similar.

Table 2 presents the initial and final moisture content and COD concentrations measured from the surface of units in Trial 2. Once again, lack of replication of the initial values limits the certainty of the calculated amount of moisture and COD loss. As observed in trial 1, there was no significant difference between the final moisture content and COD concentration measured in any of the treatments and the control. This may be due to the insensitivity of the tests for complex heterogeneous material, but certainly does not support any hypothesis that treatment of pit-latrine contents with commercial pit-latrine additives has any measurable effect on the biological activity in the pit-latrine material.

\section{Discussion}

Two trials were performed to test the hypothesis that the use of commercial pit-latrine additives could accelerate the rate of mass loss in pit-latrine samples, or the rate of biological activity in pit-latrine samples. It was shown that the data obtained in these trials did not support either of these hypotheses. It was observed that the rate of mass loss and biological activity in pit-latrine samples were significant under aerobic conditions without the assistance of commercial additives, while no statistically verifiable effect of treatment with a range of these additives could be observed on mass loss and biological activity rates.

These findings are to a certain extent in contradiction of findings of other studies into pit-latrine additives (Jere et al., 1998; Taljaard et al., 2003). However, unlike (Taljaard et al., 2003), this study was designed to test the commercial additives at the dosage rate $\left[\mathrm{g} / \mathrm{m}^{2}\right.$ surface area] at which they would normally be applied, and unlike Jere et al. (1998) using the same application technique as would normally be applied.

\section{Effect of sample heterogeneity}

Although pit-latrine content samples should be well mixed before being used in pit-latrine additive trials, a random distribution in biodegradation and mass loss rates in sub-samples was expected due to the slightly different nature of the material in each sub-sample. Consequently, differences in mass loss rate were expected between individual test units. Individual units 
exhibiting high mass loss rates therefore do not indicate that the treatment applied for that particular unit was responsible for increasing mass loss rate; the conclusion that a treatment has a significant effect on degradation or mass loss rates observed in control or reference treatments had necessarily to be made on the basis of a systematic change observed in a number of replicates of the same treatment.

\section{Micro-organism load in commercial additives vs. pit contents}

In order to explain the results obtained in this study, it was hypothesised that commercial pit-latrine additives consisting of preserved micro-organisms capable of degrading organic material are able to digest organic material found in pit-latrine contents, but that the number of active micro-organisms dosed to the pit in an application of a commercial pit-latrine additive is orders of magnitude less than the number already present in the pit-latrine contents. This is in line with the findings of Buckley et al. (2008) that significant degradation occurs in the top (aerobic) layer of the pit latrine due to the activity of micro-organisms that originate from faeces, the soil and any additional organic material that may be added to the pit latrine.

To test this hypothesis, samples of pit-latrine additive and pit-latrine contents were cultured on a nutrient agar medium. As this was only a rough initial test, results obtained are not statistically verifiable. However, observations indicated that the plate-able micro-organism concentration per gram of pitlatrine contents was of a similar order of magnitude to the concentration per gram of commercial pit-latrine additives. If these results are correct, they imply that, in order to achieve a $50 \%$ increase in the rate of degradation in the surface of the pit-latrine contents, a mass of commercial pit-latrine additive of $50 \%$ of the mass of VIP solids in the pit surface layer must be added. For a pit with a surface area of approximately $1.4 \mathrm{~m}^{2}$ and assuming the surface layer can be taken to be between 20 and $50 \mathrm{~mm}$ deep, the volume of pit-latrine contents in the surface layer can be calculated to be between 28 and $70 \ell$, i.e. probably between 30 and $80 \mathrm{~kg}$. Thus between 15 and $40 \mathrm{~kg}$ of additive (on a dry basis in their present formulation) would have to be supplied to the pit on a regular basis to ensure that the degradation rate remained $50 \%$ higher than that which would occur anyway. The cost of this treatment, and the additional volume that it would add to the pit contents would seem to prohibit this approach.

\section{Fate of additives beneath the surface layer}

Buckley et al. (2008) proposed that the mechanism of degradation that occurs in a pit is a natural one in which a significant portion of biodegradable material is aerobically degraded by micro-organisms already present in faeces and soil while it resides on the surface of the pit. When the material in question is covered over, the rate of degradation drops due to a reduction in the availability of oxygen to micro-organisms. Thereafter, a slow process of anaerobic digestion results in further degradation of remaining biodegradable material. After a certain residence time in the pit, it is hypothesised that virtually all biodegradable material will have been converted to biogas or non-degradable solids, and what remains in the lower levels of the pit contents is biologically inert solids.

The fate of micro-organisms added as part of a commercial pit additive formulation would be similar to that of a naturally occurring micro-organism in that, once they were covered over, limitation of oxygen supply would result in the activity of the micro-organisms dropping dramatically.

Preliminary results from a Water Research Commission project that is in progress at the time of writing this paper (WRC Project No. K5/1745 "After the pit is full then what? Strategies to manage on-site dry sanitation systems into the future") indicate that there is very little biodegradable material left in the lower layers of material accumulated in a pit latrine at the time of pit exhumation. These results imply that commercial pit-latrine additives that consist of micro-organisms can have very little effect on this material, and thus call into question the claims that pit-latrine additives can result in the complete reduction of pitlatrine contents with consistent use.

\section{Conclusions}

Two trials of the laboratory-scale pit-latrine additive testing protocols were undertaken using commercial pit-latrine additives. The following general conclusions were drawn from the results of these trials:

(Untreated) pit-latrine contents are extremely heterogeneous, varying in composition, biodegradability and biological activity, both within samples from a single pit, between different layers within the same pit, and between different pits. Therefore, there is a distribution of rates of biological activity and dehydration, which may be expected to be found in any trial, whether on a laboratory or a field trial scale. Thus, a treatment may only be considered to have had an affect on these rates if it can be shown that there is a systematic and statistically significant change in the rate of mass loss or rate of biological activity in the pit-latrine contents as a result of the treatment. This study found that:

- Treatment of pit-latrine contents with commercial pit-latrine additive products had no statistically significant effect on the rate of mass loss of pit-latrine contents under aerobic or anaerobic conditions

- There was no discernable difference in the final moisture content and final COD concentration in the surface of test units between treatments and controls in either of the trials, although there was a difference between the two trials. The difference was largely ascribed to the reduced dehydration rate in Trial 2 as a result of increased relative humidity in the fume hood. Thus there is no evidence that the rate of COD or moisture removal was accelerated by treatment with commercial pit-latrine additives.

- Although overall mass loss rates were similar between the two trials, the rate of moisture loss in Trial 2 was lower than in Trial 1 due to increased relative humidity in the fume cupboard. This implies that the rate of mass loss through aerobic biological activity was higher in Trial 2 than in Trial 1. This may be attributed to the differences in composition of the pit-latrine contents used in the two trials, but may also have been affected by the fact that samples did not dehydrate to the same extent in Trial 2 as they did in Trial 1.

Based on these results, it was concluded that treatment with commercial pit-latrine additives was not able to accelerate the rate of biodegradation or mass loss within pit-latrine contents.

\section{Acknowledgements}

This research was funded by the Water Research Commission. The research was made possible with the assistance of eThekwini Water and Sanitation Services, UWP engineers and Fukamela Contractors. The authors would like to gratefully acknowledge 
the participation of CF Nwaneri, BF Bakare, E Balboni and D Magagna.

\section{References}

BESTER JW and AUSTIN LM (2000) Design, Construction, Operation and Maintenance of Ventilated Improved Pit Toilets in South Africa. WRC Report No. 709/1/00 ISBN 186845647 1. Water Research Commission, Pretoria, South Africa.

BUCKLEY CA, FOXON KM, BROUCKAERT CJ, RODDA N, NWANERI C, BALBONI E, COUDERC A and MAGAGNA D (2008) Scientific Support for the Design and Operation of Ventilated Improved Pit Latrines (VIPS) and The Efficacy of Pit Latrine Additives. WRC Report No. TT 357/08, ISBN 978-1-77005-718-0. Water Research Commission, Pretoria, South Africa.

COTTON A, FRANCEYS R, PICKFORD J and SAYWELL D (1995) On-Plot Sanitation in Low-Income Urban Communities: A Review
Of Literature. WEDC, Loughborough University of Technology, ISBN 090605544 X. Water Engineering and Development Centre, Leicestershire UK.

FRANCEYS R, PICKFORD J and REED R (1992) A Guide to the Development of On-Site Sanitation. WHO, Geneva. http://www. who.int/water_sanitation_health/hygiene/envsan/onsitesan.pdf (Accessed 5 June 2007)

JERE $M$, CHIDAVAENZI $M$, NHANDARA $C$ and BRADLEY $M$ (1998) The effect of non-pathogenic bacteria on latrine sludge. Proc. $24^{\text {th }}$ WEDC Conf.: Sanitation and Water for All. 31 August - 24 September, Islamabad, Pakistan.

STILL DA (2002) After the pit latrine is full....What then? Effective options for pit latrine management. Proc. WISA Bienn. Conf. 19-23 May 2002, Durban, South Africa.

TALJAARD L, VENTER A and GORTON D (2003) Evaluation of Different Commercial Microbial or Microbially-Derived Products for the Treatment of Organic Waste in Pit Latrines. WRC Report No. 1377/1/05. Water Research Commission, Pretoria, South Africa. 